\title{
Stabilization of Chitosan-Based Polyelectrolyte Nanoparticle Cargo Delivery Biomaterials by a Multiple Ionic Cross-Linking Strategy
}

\author{
Marjan Motieia ${ }^{a^{*}}$,Vladimír Sedlařík ${ }^{a}$, Lucian A. Lucia ${ }^{b, c}$, Haojie Fei ${ }^{a}$, Lukášs Münster ${ }^{a}$ \\ ${ }^{a}$ Centre of Polymer Systems, Tomas Bata University in Zlín, Třida Tomáše Bati 5678, 76001 Zlín, Czech Republic \\ ${ }^{b}$ Departments of Forest Biomaterials, Chemistry, Campus Boxes 8005, 8204, North Carolina State University, \\ Raleigh, North Carolina 27695, United States \\ 'State Key Laboratory of Bio-based Materials \& Green Papermaking, Qilu University of Technology / Shandong \\ Academy of Sciences, Jinan, PR China 250353 \\ Email:motiei@utb.cz.
}

\begin{abstract}
PolyElectrolyte Nanoparticles (PENs) obtained by layer-by-layer self-assembly of polycations/polyanions suffer from a lack of colloidal stability in physiological conditions. We report a simple innovative approach for increasing their stability by multiple ionic cross-linkers. Herein, a chitosan-based core was stabilized by polyanions such as tripolyphosphate and dextran sulfate at pHs of 3 (aPENs) and 8 (bPENs) to improve the quality of electrostatic interactions in the core and manage self-assembly of polyethyleneimine shell onto the core. The physicochemical properties of the particles were characterized by DLS, SEM, TEM, FT-IR, and TGA. TEM micrographs showed visible core/shell structures of bPENs. From particle size and polydispersity indices, the bPENs stability was salt concentration-dependent. The release profiles of PENs using nicotinic acid demonstrated sustained release in a $\mathrm{pH}$-independent manner with a good fit of Korsmeyer-Peppas model. These results suggest that multiple ionic cross-linkers can be an efficient approach to increase the colloidal stability of PENs.
\end{abstract}

Keywords: Polyelectrolyte Nanoparticles, Colloidal Stability, Chitosan, Polyethyleneimine, Tripolyphosphate, Dextran Sulfate. 


\section{Introduction}

Currently, PENs have attracted more attention due to strong self-assembly of reversible electrostatic interactions between oppositely charged polymers useful for encapsulation and controlled release of charged cargos. These complexes exhibit desirable physicochemical properties of various polymers and possess the advantage of simple preparation. Nevertheless, PENs suffer from a lack of stability in physiological conditions due to various characteristics of building components (i.e., concentration and $\mathrm{MW}$ ) and environmental conditions (i.e., pH, temperature, and especially ionic strength) (Wu \& Delair, 2015). Several researchers have demonstrated the physical stability of PENs consisting of chitosan (CS)/hyaluronan (Umerska et al., 2012), CS/poly- $\gamma$-glutamic acid (Lin et al., 2005), Carboxymethyl-trimethyl CS/chondroitin sulfate (Hansson et al., 2012) and CS/acylated cruciferin (F. Wang, Yang, Ju, Udenigwe, \& He, 2018) in water for different periods. Nonetheless, stability improvements in physiological conditions needed suppressing the deleterious effect of salts by various processing techniques including steric stabilization (Costalat, David, \& Delair, 2014), zinc ion coordination (Wu \& Delair, 2015), thiolation (Verheul et al., 2011), and quaternization (Bal et al., 2010) of the original polymers. However, sterically stabilized nanoparticles lose the intrinsically appealing polyelectrolyte property of bulk fluidity as a function of $\mathrm{pH}$, temperature, and ions; ion coordination, thiolation, and quaternization also need chemical reagents or organic solvents. To overcome this handicap, our current hypothesis suggests using multiple ionic cross-linkers as a novel simple approach, and devotes to evaluate the effectiveness of multiple cross-linkers and $\mathrm{pH}$ on the stability of the carbohydrate-based nanoparticles.

CS is a biocompatible, biodegradable, and antibacterial chitin derivative composed of randomly distributed $\beta$-1,4-linked D-glucosamine and N-acetyl-D-glucosamine units. CS can form complexes with polyanions such as tripolyphosphate (TTP) and dextran sulfate (DS) due to protonation of amino groups below its pKa (6.5) (Motiei \& Kashanian, 2017). Ionic gelation of CS with TTP as a nontoxic, weak polyprotic acid, and multivalent anionic cross-linker improves the quality of electrostatic interactions (Diop et al., 2015). DS is a biodegradable, biocompatible, sulfated, and branched polysaccharide that is expected to show strong ionic interactions through the positive ammonium groups of CS, increase the stability of the nanoparticles, and be deployable in drug delivery systems (Quiñones, Peniche, \& Peniche, 2018). 
Polyethyleneimine (PEI) is composed of amino groups that is partially protonated at physiological and endosomal pHs, and is able to induce endosomal rupture by its unique "proton sponge effect". It consequently facilitates the polyplexes release into cytosols (W. Wang et al., 2017). However, the cytotoxicity issue of high MW PEI has been addressed by modifying PEI with numerous hydrophilic or hydrophobic segments (Liu et al., 2015), assembling with other degradable and biocompatible polymers, and/or using low MW PEI (W. Wang et al., 2017).

Overall, a chief innovation of the present work was the simple design and fabrication of stable nanoparticles to resist the destabilizing environment of living tissues (i.e., $\mathrm{pH}$ and ionic fluctuations). These nanosystems were composed of an inner CS core, synthesized by an ionic gelation technique using polyanionic cross-linkers at two different $\mathrm{pHs}$ and a polycationic PEI shell. The optimized formula were fully characterized by DLS, SEM, TEM, FT-IR, and TGA. Furthermore, their effectiveness on physical stability was evaluated by DLS and SEM after storage in PBS $(0,1$, and $10 \mathrm{mM})$ at $4{ }^{\circ} \mathrm{C}$. Finally, nicotinic acid (INN) was used as a hydrophilic model drug for evaluation and comparison of drug loading, in vitro drug release as well as kinetics modeling of these PENs.

\section{Experimental section}

\subsection{Materials}

Low molecular weight CS with MW of 50-190 kDa, degree of deacetylation $\geq 75 \%$, and degree of substitution=1.26 (Supplementary information, SI) was provided by Sigma Chemical Co. (St. Louis, MO, USA). DS sodium salt (MW of 7-20 kDa, sulfur content 17-19\%), PEI (average MW $1.3 \mathrm{kDa}$ by LS, concentration 48-52\%), TPP, INN, acetic acid, sodium chloride, disodium hydrogen phosphate, potassium chloride, potassium dihydrogen phosphate and dialysis tubing with cut off 12 kD MWCO were also purchased from Sigma Chemical Co.

2.2. Preparation of PENs at different $\mathrm{pHs}$ with ionic cross-linkers 
To optimize the formulation, CS was dissolved in $1 \%$ acetic acid solution adjusting to $\mathrm{pH} 5$, while stirring in the presence of $0.5 \%$ Tween 20 as a nonionic steric stabilizer (500 rpm, 60 minutes, 45$\left.50{ }^{\circ} \mathrm{C}\right)$. Afterward, different volumes of DS (1mg/mL, $\mathrm{pH} 3$ and $\mathrm{pH}$ 8) were added to the solution in constant TPP concentration $(2 \mathrm{~mL}, 1 \mathrm{mg} / \mathrm{mL}, \mathrm{pH} 3$ and $\mathrm{pH}$ 8) under stirring (500 rpm, 10 minutes) to obtain the smallest z-average size of the core. Finally, an optimized PEI concentration was determined by mixing different PEI volumes $(10 \mathrm{mg} / \mathrm{mL}, \mathrm{pH} 8)$ with the core solution under stirring (500 rpm, 10 minutes). The final optimized formula were used for other characterizations. In INN loaded PENs, INN was added to the CS solution under gentle stirring at room temperature for 2 hours before incorporation in the cross-linkers. After addition of cross-linkers and PEI shell, the prepared samples were utilized for evaluation of z-average size, zeta $(\zeta)$-potential, and in vitro drug release studies.

\subsection{Characterization of PENs}

DLS (model 3600, Malvern Instruments Ltd., Worcestershire, UK) was used for evaluation of $\zeta$ potential and particle size at $25^{\circ} \mathrm{C}$ by disposable polystyrene cuvettes. Morphology of the dried nanoparticle suspension was observed by SEM (Nova 450 NanoSEM, FEI, Brno, Czech Republic) and TEM (JEM 2100, JEOL Ltd., Japan) operated at $5.00 \mathrm{kV}$ and $160 \mathrm{kV}$ accelerating voltage, respectively. For SEM, the dried suspension on a piece of aluminum foil was placed on the SEM specimen stub with a double-sided carbon adhesive disc (Taab, Berkshire, UK) and sputter-coated with gold/palladium (SC7620 Mini Sputter Coater, Quorum Technologies,10mA for 45 seconds), and TEM sample preparation was done by a dried suspension on a carbon-coated 300-mesh copper grid (Structure Probe Inc., West Chester, USA). The physical stability of nanoparticles in PBS (0, 1, and $10 \mathrm{mM}, \mathrm{pH} 7.4$ ) at $4{ }^{\circ} \mathrm{C}$ was monitored by SEM after two weeks and DLS at predetermined time intervals for two months. For this purpose, one volume of the particles was mixed with the same volume of PBS $(2 \times)$ to obtain PBS concentrations of 1 and $10 \mathrm{mM}$. Freeze-dried samples were used for further assays, including FT-IR and TGA analysis. Frozen samples in liquid nitrogen were lyophilized using a Freeze Dryer (SCANVAC Coolsafe ${ }^{\mathrm{TM}}$, model 110-4, Denmark), condenser temperature $-110^{\circ} \mathrm{C}$, pressure 0.2 mbar. FT-IR spectra were carried out with a Nicolet iS5 spectrometer at 64 scans and a resolution of $4 \mathrm{~cm}^{-1}$ over a wavenumber range of 4000-400 $\mathrm{cm}^{-1}$. The thermogravimetric analysis was performed on a TA Instruments Q500 
Thermogravimetric Analyzer at $10^{\circ} \mathrm{C} / \mathrm{min}$ heating rate under the nitrogen atmosphere from 25 to $700^{\circ} \mathrm{C}$, and then weight loss percentage of the components was calculated using the Universal Analysis 2000 system.

\subsection{Drug loading and in vitro release assays of PENs}

Drug loading and in vitro release of INN loaded-PENs were evaluated by dialysis technique and in the presence of a non-toxic and isotonic release medium, PBS. For evaluation of encapsulation efficiency (EE) and loading capacity (LC) of INN loaded-PENs, the dispersions ( $10 \mathrm{~mL})$ were placed into $100 \mathrm{~mL}$ PBS10 mM, pH 7.4 by a dialysis tube and then the entire system was kept in an orbital incubator (Stuart SI500, UK) at $37 \pm 0.5^{\circ} \mathrm{C}, 40 \mathrm{rpm}$ for 1 hour. EE (\%) and LC (\%) were calculated according to Eqs. (1) and (2), respectively (Motiei, Kashanian, Lucia, \& Khazaei, 2017), where Total INN was the amount of primary INN added to the solution and Free INN was assessed through UV-vis spectrophotometry (CARY 300 Conc, USA) at $261 \mathrm{~nm}$ versus a calibration curve $\left(\mathrm{R}^{2}=0.999, \mathrm{n}=3\right)$.

Eq. (1) $\quad$ EE $(\%)=\frac{\text { Total INN-Free INN }}{\text { Total INN }} \times 100$

Eq. (2) $\quad$ LC $(\%)=\frac{\text { Total INN-Free INN }}{\text { Nanoparticle Weights }} \times 100$

Afterward, for evaluation of the in vitro release rate, the medium was replaced with $50 \mathrm{~mL}$ freshly PBS $10 \mathrm{mM}$ (pH 7.4 and 3), and the entire system was kept in an orbital incubator at $37 \pm 0.5^{\circ} \mathrm{C}$, $40 \mathrm{rpm}$. At pre-determined time intervals, $3 \mathrm{~mL}$ of the medium was taken, and the same volume of the fresh medium was replaced in the system. The amount of INN in the medium was evaluated by UV-Vis spectrophotometry versus a calibration curve at $261 \mathrm{~nm}$.

\subsection{Statistical analysis}

All experiments were done in triplicates, and the data were presented as mean \pm standard deviation. Statistical analysis was carried out using Microsoft ${ }^{\circledR}$ Excel and SPSS® software. A Student's ttest was used for comparison of mean data, and the differences were considered to be significant at the value of $\mathrm{P}<0.05$. 


\section{Result and discussion}

\subsection{Evaluation of z-average size and $\zeta$-potential of PENs}

The effect of ionic cross-linkers' $\mathrm{pH}$ on the size distribution and $\zeta$-potential of PENs was evaluated, because $\mathrm{pH}$ is one of the main parameters affects the ionization degree of functional groups, the ionic interactions among the components, and consequently the particle size and $\zeta$ potential (Motiei, Kashanian, Lucia, et al., 2017). The smallest size and relatively narrow distribution of aPENs $(268.8 \pm 4.00 \mathrm{~nm}$ and $0.40 \pm 0.01)$ and bPENs $(2 \mathrm{f} 46.97 \pm 0.25 \mathrm{~nm}$ and $0.35 \pm 0.03$ ) were observed at the optimal concentration of DS in the range of $0.133 \mathrm{mg} / \mathrm{ml}$ (Fig. 1a and Table S1). It was expected that bPENs showed larger size distributions than aPENs due to the dissociation of TPP into $\mathrm{OH}^{-}$and tripolyphosphoric ions, production of coacervation barrier by $\mathrm{OH}^{-}$, and low diffusion of $\mathrm{P}_{3} \mathrm{O}_{10}{ }^{5-}$ into the core to weakly overcome the repulsive forces between protonated amino groups of CS chains (Motiei \& Kashanian, 2017). Nonetheless, this study showed conflicting evidence regarding the effect of DS on the size distribution of PENs.

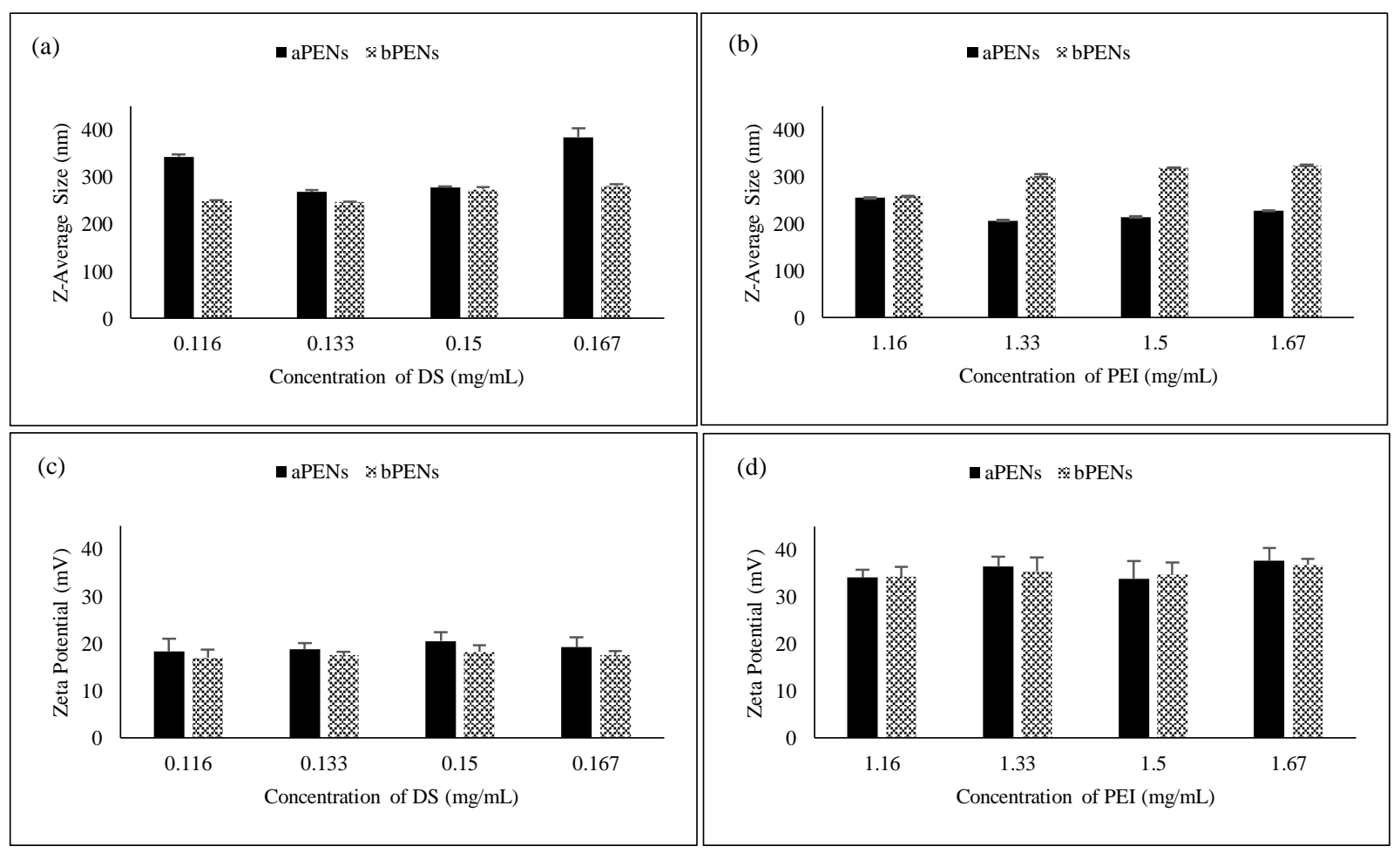


Fig. 1. The relationship between z-average size ( $\mathrm{a}$ and $\mathrm{b}$ ) and $\zeta$-potential ( $\mathrm{c}$ and d) of PENs to concentration and $\mathrm{pH}$ of DS and PEI.

In the next stage, the addition of PEI at $\mathrm{pH} 8$ increased the size of bPENs while at $\mathrm{pH} 3$, aPENs showed significant size reduction (Fig. 1b and Table S1). The smallest hydrodynamic diameter and PDI of aPENs (206.40 $\pm 2.09 \mathrm{~nm}$ and $0.29 \pm 0.01)$ and bPENs $(258.13 \pm 4.85 \mathrm{~nm}$ and $0.25 \pm 0.01)$ were found at PEI concentration of $1.33 \mathrm{mg} / \mathrm{mL}$ and $1.16 \mathrm{mg} / \mathrm{mL}$, respectively, whose z-average size differences between the two groups were considered statistically significant. $\mathrm{pH}$-sensitivity of PENs' size can be explained by inter/intramolecular interactions among the polyelectrolytes having different pKa values (i.e., CS, TPP, DS, and PEI are close to 6.5, 0.89, $<2$ and 7-10, respectively). At low $\mathrm{pH}$, only $\mathrm{P}_{3} \mathrm{O}_{10}{ }^{5-}$ anions are found in TPP solution, while highly protonated CS will be partially ionic cross-linked due to an insufficient concentration of TPP (Ajun, Yan, Li, \& Huili, 2009). Herein, owing to using multiple cross-linkers, TPP was utilized at constant concentration until DS completed the deficiency of TPP concentration. Thereafter, negatively charged sulfate groups of DS caused a strong electrostatic interaction with the remaining protonated amino groups of CS and also PEI, which led to more compact structures. At pH8, TPP is dissociated into $\mathrm{OH}^{-}$and TPP ions $\left(\mathrm{HP}_{3} \mathrm{O}_{10}{ }^{4-}\right.$ and $\left.\mathrm{P}_{3} \mathrm{O}_{10}{ }^{5-}\right)$ to produce a coacervation barrier by $\mathrm{OH}^{-}$and lower the diffusion of $\mathrm{P}_{3} \mathrm{O}_{10}{ }^{5-}$ into the core (Motiei \& Kashanian, 2017). Although the alkaline cross-linkers lowered the net positive charge of CS, it maintained sufficient binding sites for diffused $\mathrm{P}_{3} \mathrm{O}_{10}{ }^{5-}$. Finally, decreasing electrostatic attraction among negatively charged DS with PEI and CS and replacing with hydrogen bonds lead to a decrease in electrostatic attraction and a significant increase in particle size (H. Wang, Yang, \& Sun, 2017). A representation is in Fig. 2. 


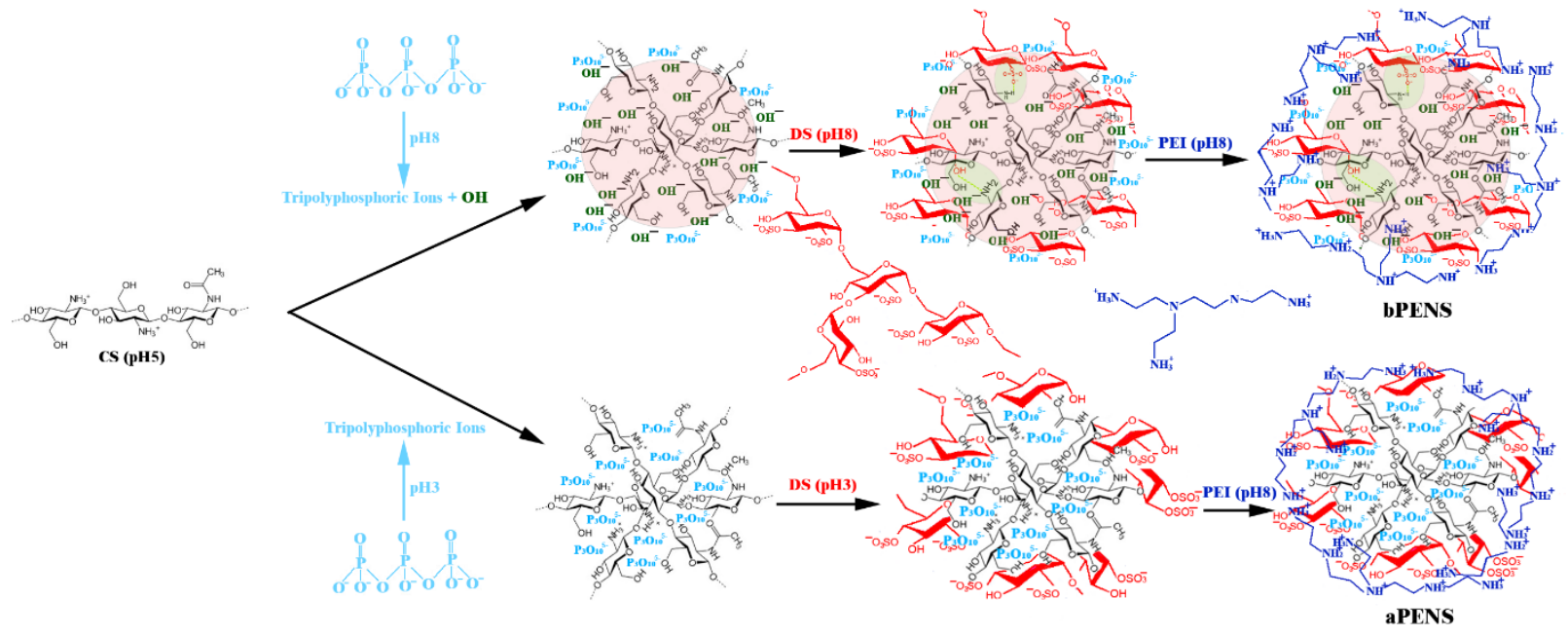

Fig. 2. A proposed scheme of PENs formation at different $\mathrm{pHs}$ of cross-linkers.

$\zeta$-potential is another important physicochemical parameter that critically influences the nanoparticle stability by repulsive forces. According to Fig. 1 (c and d) and Table S1, $\tau \eta \varepsilon \zeta-$ potential of these two groups in the presence and absence of PEI was positive with statistical significance $(\mathrm{p}<0.05)$. Therefore, in the absence of PEI, DS could not shield the entire positive charges of CS on the particles surface, and addition of PEI led to more amino groups and higher positive charge. However, the $\mathrm{pH}$ of cross-linkers did not influence the surface charge of the particles because $\tau \eta \varepsilon \zeta$ potential of aPENs and bPENs showed no significant difference.

In addition, the physicochemical characteristics of INN loading aPENs and bPENs were evaluated and summarized in Table 1. Considering different synthesis conditions, no significant difference was found between the mean diameters of loaded and unloaded nanoparticles in each group. PDI values below 0.3 with no significant difference also indicated monodisperse distributions of INN loaded and unloaded PENs. However, the $\zeta$-potential of aPENs decreased significantly after the addition of INN ( $p<0.05)$ as opposed to bPENs. aPENs due to their compact structure were not able to entrap the whole amount of INN available in the solution; thus, several could adsorb on their surfaces and decrease surface charge. Nonetheless, bPENs entrapped INN within the particles' core with no significant effect on surface charge. 
Table 1. Physicochemical characterization of PENs before and after loading INN

\begin{tabular}{lllll}
\hline & \multicolumn{2}{l}{ aPENs } & \multicolumn{2}{l}{ bPENs } \\
\cline { 2 - 5 } & Unloading INN & Loading INN & Unloading INN & Loading INN \\
\cline { 2 - 5 } z-average size $(\mathrm{nm})$ & $204.40 \pm 0.78$ & $211.37 \pm 1.68$ & $254.40 \pm 0.62$ & $250.10 \pm 0.36$ \\
PDI & $0.26 \pm 0.01$ & $0.28 \pm 0.01$ & $0.25 \pm 0.00$ & $0.26 \pm 0.01$ \\
$\zeta$-potential & $34.87 \pm 2.66^{*}$ & $23.6 \pm 1.54 *$ & $33.83 \pm 4.60$ & $34.23 \pm 3.25$ \\
\hline$* p$ value $<0.05$ & & & &
\end{tabular}

\subsection{Morphology of the particles}

Fig. 3 indicated SEM and TEM micrographs of PENs, which confirmed uniform dispersed spherical particles with a smaller size in acidic condition than basic one. SEM images provided the surface morphology and accurate size of aPENs (Fig.3a) and bPENs (Fig.3b) of 141.20 $\pm 65.57 \mathrm{~nm}$ and 204.23 $\pm 18.38 \mathrm{~nm}$, respectively. TEM images confirmed the core-shell nanostructure of bPENs (Fig.3d), where a dark ring of PEI coated CS-based core $(86.93 \pm 8.00 \mathrm{~nm})$, and the total size was $\sim 166.54 \pm 14.05 \mathrm{~nm}$. Nonetheless, this core/shell structure was not obvious in aPENs (Fig.3c) with a size of $117.30 \pm 26.70 \mathrm{~nm}$, which might be attributed to completely crosslinked CS by the polyanionic cross-linkers and strong electrostatic attractions among negatively charged DS and protonated amino groups of PEI shell. Nonetheless, at $\mathrm{pH} 8$, the presence of a coacervation barrier, partially electrostatic interactions, and hydrogen bonding led to visible core/shell structure. 


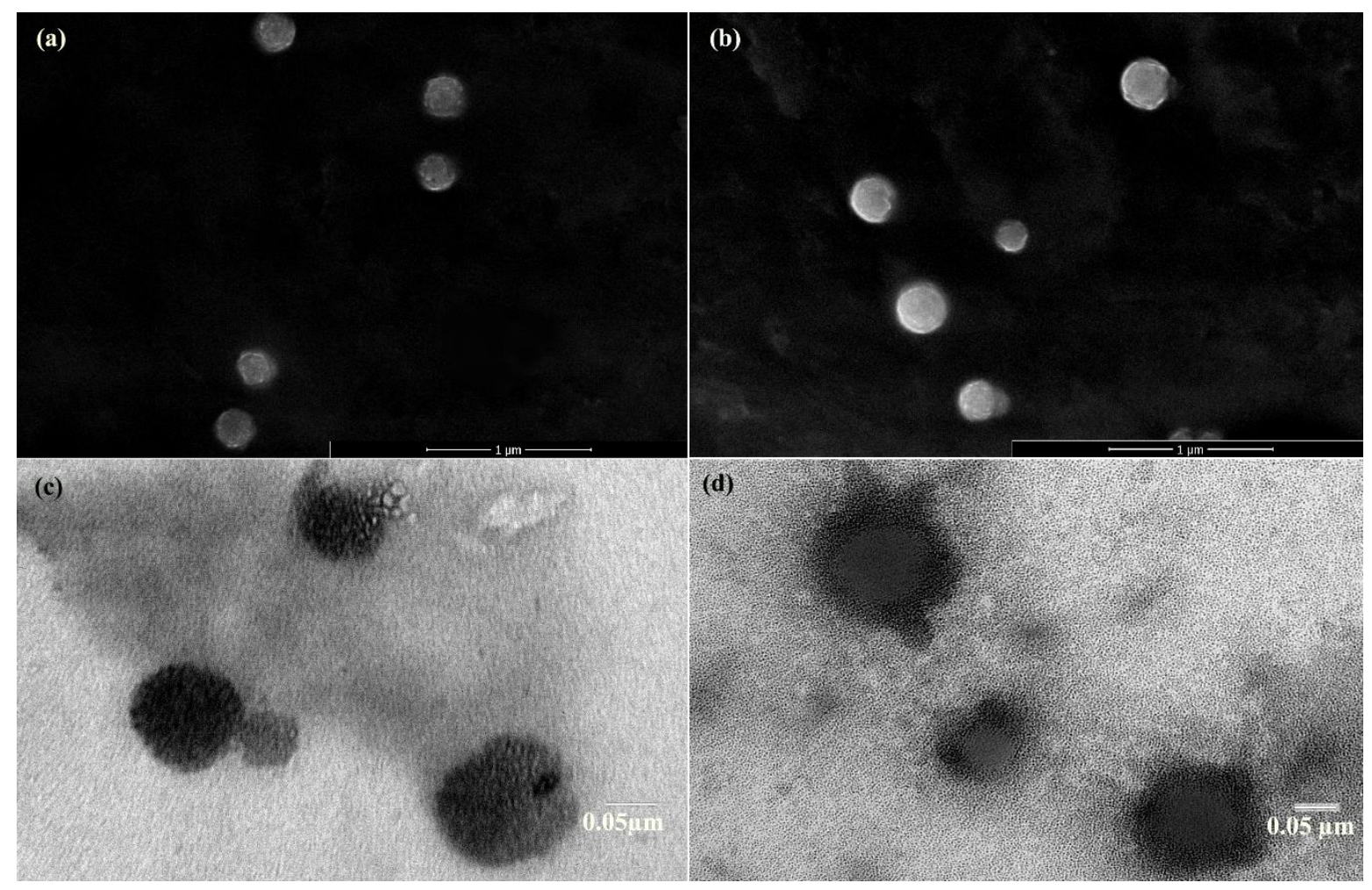

Fig. 3. Comparing SEM and TEM images of aPENs and bPENs. SEM images of (a) aPENs and (b) bPENs and TEM images of (c) aPENs and (d) bPENs in a dried state.

\subsection{Evaluation of colloidal stability of PENs}

PENs indicated high sensitivity to the characteristics of the components and environment (Wu \& Delair, 2015), as using lower MW CS featured decreased stability in water due to less inter/intramolecular hydrogen bonding, and the dispersions obtained with CS (degree of acetylation 4-48\%) were destabilized or completely solubilized upon dilution in PBS (Wu \& Delair, 2015). Herein, low MW CS (degree of acetylation 15-25\%) was stabilized by two anionic cross-linkers (TPP/DS), coated with a PEI shell, and then evaluated stability by DLS at three salt concentrations (0, 1 and $10 \mathrm{mM}, \mathrm{pH}$ 7.4) upon storage at $4{ }^{\circ} \mathrm{C}$ for two months. All samples presented slight increases in size with no aggregates confirmed by PDI values of $0.22 \pm 0.04$ and $0.21 \pm 0.04$ in aPENs and bPENs, respectively. Z-average size of aPENs showed a significant increase from $176.31 \pm 8.47$ to $352.43 \pm 3.35$, and bPENs from $219.42 \pm 15.54$ to $284.80 \pm 4.41$ after storage in water for 2 months (Table S2). It can be deduced that the presence of TPP generates an inflow of water by osmosis to equalize the solute concentrations between the two sides, which 
leads to increase the size significantly in absence of salt (Rampino, Borgogna, Blasi, Bellich, \& Cesàro, 2013). It was also confirmed by SEM images after the storage of PENs in water for 2 weeks (Fig. S1b and f). SEM images of PENs showed a significant increase in particle size, and the presence of smaller particles with sizes $<50 \mathrm{~nm}$ likely belonged to separated PEI fractions after core swelling.

When the particles were stored in PBS $1 \mathrm{mM}$, the z-average size of aPENs also increased significantly to $239.47 \pm 3.04$, and bPENs to $267.7 \pm 6.17$ (Table S2). Nonetheless, after storage in PBS $10 \mathrm{mM}$, the hydrodynamic diameter of aPENs changed to $245.5 \pm 5.54$, and bPENs to $243.83 \pm 8.70$, which indicated higher salt stability of bPENs compared to aPENs. Fig. S1 has also demonstrated this phenomenon after two weeks storage in PBS 1 and $10 \mathrm{mM}$. Using the SEM micrographs as a guide, increased size and delamination of the aPENs' shell were particularly obvious in PBS 1mM (Fig. S1c), but bPENs, however, indicated compact structures with smooth surfaces and no delamination after two weeks (Fig. S1g and h). Considering these features, bPENs showed increased stability in the presence of salts, and higher salt concentration led to more stabilization, in agreement with Jonassen et al. (Jonassen, Kjøniksen, \& Hiorth, 2012). On the other hand, $\mathrm{Wu}$ and Delair showed precipitation of PENs in the presence of $3.0 \mathrm{mM}$ salt $(\mathrm{Wu} \&$ Delair, 2015).

According to Fig. 4, the size variations of bPENs were negligible, while several fluctuations were observed in aPENs. These fluctuations can be attributed to inter/intramolecular interactions because the high positive charge of amino groups will cause strong attractive electrostatic interactions with negatively counter-ions. With regards to the neutralization of electrostatic interactions, salt ions can lead to the passive osmotic diffusion of $\mathrm{H}_{2} \mathrm{O}$, inducing hydrogen bonding and increasing size. Nonetheless, the stability of the particles is attributed to the small size and narrow particle size distribution (Fan, Yan, $\mathrm{Xu}, \& \mathrm{Ni}, 2012$ ), so the particles tend to excrete extra water and contract in size. However, $\mathrm{pH} 8$ led to deprotonation of amino groups, lowering of electrostatic attractions and partial hydrogen bonding displacement, which improves the stability of PENs more than the acidic situations due to the type of inter/intramolecular forces. 
(a)

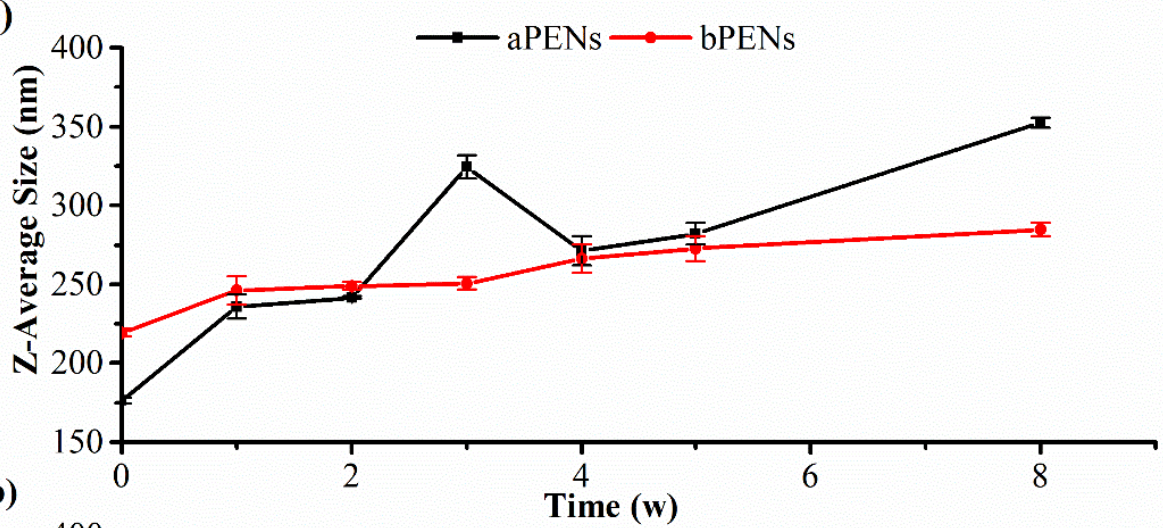

(b)
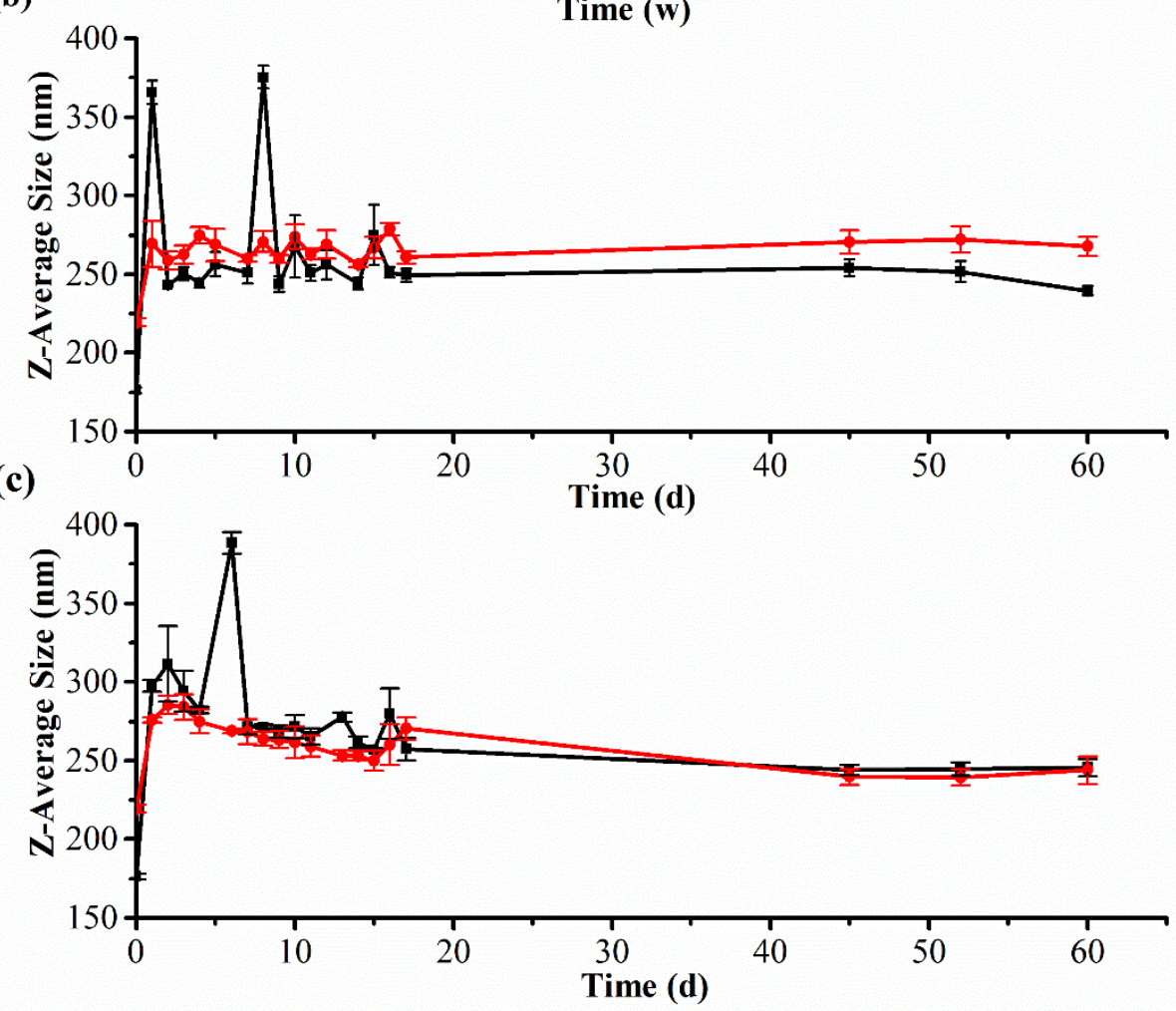

Fig. 4. Stability studies of PENs' dispersions in water (a), PBS $1 \mathrm{mM}(\mathrm{b})$, and PBS $10 \mathrm{mM}$ (c) at $4{ }^{\circ} \mathrm{C}$ for two months. 


\subsection{Infrared spectrophotometry analysis}

An appropriate approach for confirmation of intermolecular interactions is through the comparison of the FTIR spectra of the neat samples with products. According to Fig. 5, IR spectra of CS showed characteristic peaks assigned to $899 \mathrm{~cm}^{-1}$ (pyranose ring), $3373 \mathrm{~cm}^{-1}$ (OH and $\mathrm{NH}_{2}$ stretching), $2881 \mathrm{~cm}^{-1}$ (-CH stretching), $1657 \mathrm{~cm}^{-1}$ (amide I), $1595 \mathrm{~cm}^{-1}$ (amide II), $1381 \mathrm{~cm}^{-1}$ $\left(\mathrm{CH}_{3}\right.$ symmetrical deformation mode), and $1070 \mathrm{~cm}^{-1}$ (asymmetric $\mathrm{C}-\mathrm{O}-\mathrm{C}$ stretching) (Motiei, Kashanian, \& Taherpour, 2017). Pure DS spectrum demonstrated several identical absorption bands including asymmetric and symmetric $\mathrm{SOO}^{-}$stretching vibrations at $\sim 1230 \mathrm{~cm}^{-1}$ and 987 $\mathrm{cm}^{-1}$ confirmed the presence of the sulfato-group in DS spectrum, as well as the bands at about $804 \mathrm{~cm}^{-1}$ derived from asymmetric S-O-S vibration (Chavan, Bala, Pal, \& Kale, 2017). The FTIR spectrum of PEI exhibited the characteristic absorption peaks of amine groups located at 3363 $\mathrm{cm}^{-1}$ and $1647 \mathrm{~cm}^{-1}$. The other absorbance peaks showed $\mathrm{C}-\mathrm{N}$ stretching $\left(1113 \mathrm{~cm}^{-1}\right), \mathrm{C}-\mathrm{H}$ deformation $\left(1471 \mathrm{~cm}^{-1}\right), \mathrm{N}-\mathrm{H}$ deformation $\left(1608 \mathrm{~cm}^{-1}\right)$, and the stretching vibration of $\mathrm{C}-\mathrm{H}$ bonds of the alkyl chain (2954 and $2843 \mathrm{~cm}^{-1}$ ) (Yudovin-Farber, Beyth, Weiss, \& Domb, 2010). The spectra analysis of the PENs showed the peaks of $1117 \mathrm{~cm}^{-1}$ with a shoulder at $1147 \mathrm{~cm}^{-1}$, which was referred to $\mathrm{P}=\mathrm{O}$ in TPP (Motiei \& Kashanian, 2017) and formation of sulfones (Nikolić et al., 2017), respectively. aPENs showed the higher intensity of several corresponding sharp bands, including $1570 \mathrm{~cm}^{-1}$ (Amide II band), $1410 \mathrm{~cm}^{-1}$ (symmetric stretching of COO-), 924 $\mathrm{cm}^{-1}, 1014 \mathrm{~cm}^{-1}$ and $1043 \mathrm{~cm}^{-1}$ (sulfo-group) and $648 \mathrm{~cm}^{-1}$ (weak $\mathrm{N}-\mathrm{H}$ stretching vibrations). The peak at $619\left(\mathrm{O}-\mathrm{H}\right.$ bend) disappeared, while the peak at $833 \mathrm{~cm}^{-1}$ shifted to $806 \mathrm{~cm}^{-1}$ (asymmetric $\mathrm{S}-\mathrm{O}-\mathrm{S}$ vibration) in aPENs. The presence, disappearance, and shifting of these bands is attributed to polyplex formation by stronger electrostatic interactions at $\mathrm{pH} 3$. Nonetheless, at $\mathrm{pH} 8$, the presence of a few sharp peaks $~ 3421 \mathrm{~cm}^{-1}$ (primary amines), $3286 \mathrm{~cm}^{-1}$ and $3178 \mathrm{~cm}^{-1}(\mathrm{O}-\mathrm{H}(\mathrm{H}-$ bonded)) and $1637 \mathrm{~cm}^{-1}$ ( $\mathrm{C}=\mathrm{O}$ (amide I band)) can be correlated to the existence of hydrogen bindings in addition to ionic interactions. 


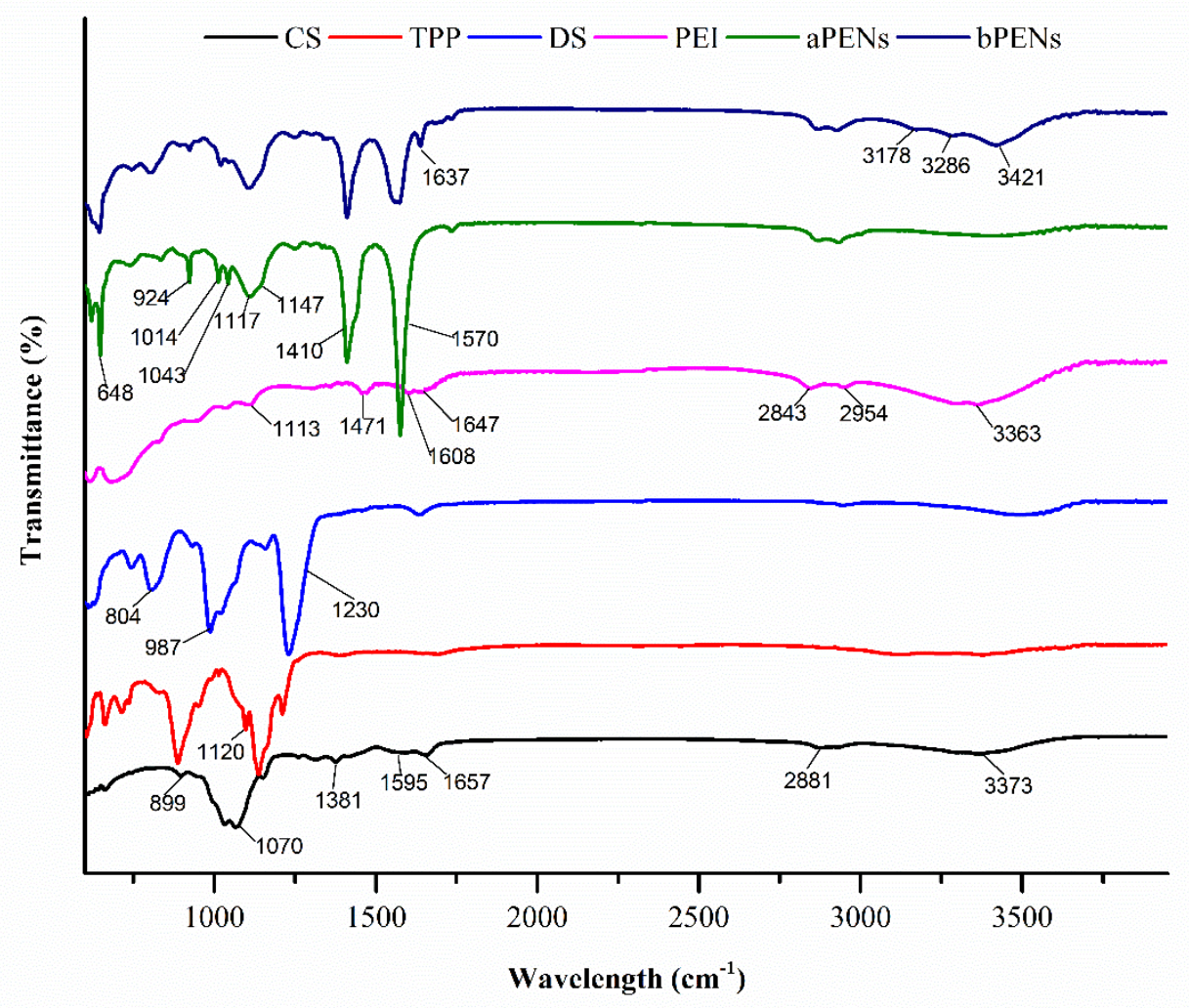

Fig. 5. FTIR spectra of PENs, i.e., aPENs and bPENs, and the neat samples, i.e., PEI, DS, TPP, and CS.

\subsection{Thermogravimetric analysis}

The thermal properties of the particles were evaluated by TGA to confirm the stability of the two particles by determining their total percentage weight loss (Table S3). Thermogravimetric analysis of all samples was performed at $25-700^{\circ} \mathrm{C}$. As shown in Fig. 6, the regular diminishment of the weights below $\sim 100{ }^{\circ} \mathrm{C}$ was mainly due to physically and chemically preadsorbed $\mathrm{CO}_{2}$, moisture, and other gases. In addition, CS and DS lost about $60 \%$ of their masses at temperatures from 225 ${ }^{\circ} \mathrm{C}$ to $525{ }^{\circ} \mathrm{C}$ due to depolymerization and loss of amino (CS), sulfate (DS), and $\mathrm{CH}_{2} \mathrm{OH}$ moieties (Can, Kavlak, ParviziKhosroshahi, \& Güner, 2018). Nonetheless, the major weight loss of PEI, which was MW-dependent occurred at 320 and $380{ }^{\circ} \mathrm{C}$ and attributed to the volatilization and decomposition of PEI molecules (Kishor \& Ghoshal, 2016). 
The thermal degradation and mainly weight loss of PENs showed four main stages. Firstly, weight loss behavior of approximately $24 \%$ of aPENs and $14 \%$ of bPENs at temperatures ranging from 140 to $300{ }^{\circ} \mathrm{C}$ was related to degradation of DS. A further increase to $400^{\circ} \mathrm{C}$ resulted in weight losses of $23.2 \%$ and $29.15 \%$ for aPENs and bPENs, respectively. The total weight loss corresponded to the degradation of CS, PEI, and freezing bound water. The third stage at $~ 300-$ $500{ }^{\circ} \mathrm{C}$ is correlated to complete degradation of DS, and the final stage with the highest degradation rate was $\sim 500-700{ }^{\circ} \mathrm{C}$ associated with the complete degradation of organic compounds. Altogether, the comparison of the total weight loss and thermal behavior of the two particles indicated that the stability of the particles is not significantly different over the temperature ranges.

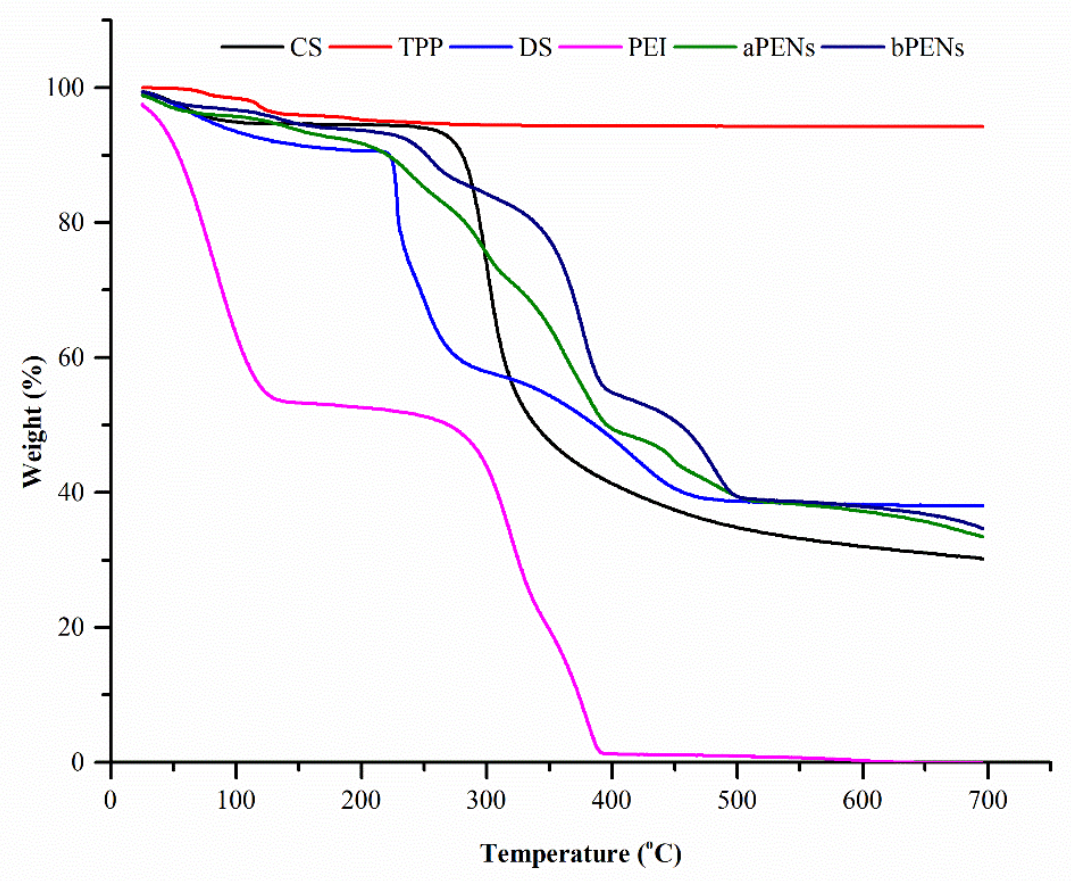

Fig. 6. Thermogravimetric analysis of PENs, i.e., aPENs and bPENs and pure compounds, i.e., CS, TPP, DS, and PEI.

\subsection{In vitro release studies in two media with different $\mathrm{pHs}$}

For illustrating the effect of cross-linkers on the in vitro release process, drug release behavior was quantified by INN in different nanostructures, including single-layer (TPP) and multilayer (TPP/DS and TPP/DS/PEI) structures. INN was selected as a model drug due to significant biochemical therapeutic roles, and its $\mathrm{pKa}$ (4.75) made it entirely anionic at the desired $\mathrm{pHs}$ 
(Gonçalves \& da Piedade, 2012). According to Table 2, different formulations illustrated approximately equal amounts of drug loading influenced by type and concentration of the polymers instead of cross-linkers $\mathrm{pH}$, because encapsulation increased in the presence of TPP/PEI more than DS, but not significantly. Lower EE in the presence of DS can be attributed to strong repulsive forces between DS chains and also DS with negatively charged INN. Nonetheless, low concentration of TPP polyanions cannot restrain the penetration of anionic INN to positively charged cores. The last layer, positively charged PEI, also increases encapsulation by strong electrostatic attractions. Table 2 also demonstrated the lowering of LC (\%) after the addition of each polyelectrolyte, which may be confirmed by strong dependency of LC on polymer weight ratio in accordance with Eq. (2).

Table 2. EE and LC of PENs composed of single-layers (TPP) to multilayer structures (TPP/DS and TPP/DS/PEI) at two different pHs of cross-linkers (3 and 8).

\begin{tabular}{lllllll}
\hline & \multicolumn{5}{c}{ aPENs } & \multicolumn{2}{l}{ bPENs } \\
\cline { 2 - 6 } & TPP & TPP/DS & TPP/DS/PEI & TPP & TPP/DS & TPP/DS/PEI \\
\cline { 2 - 7 } EE $(\%)$ & $70,16 \pm 5.10$ & $59,58 \pm 5.61$ & $71.85 \pm 2.60$ & $67,67 \pm 2.74$ & $60,64 \pm 5.47$ & $71.36 \pm 0.34$ \\
LC $(\%)$ & $22,13 \pm 1.39$ & $18,15 \pm 1.42$ & $17.38 \pm 0.62$ & $21,45 \pm 0.74$ & $18,42 \pm 1.39$ & $17.26 \pm 0.34$ \\
\hline
\end{tabular}

${ }^{*} p$ value $<0.05$

As shown in Fig. 7, in vitro release profiles can be ascribed as a two-step biphasic process including an initial burst release for $\sim 6$ hours, and a subsequent gradual release for 72 hours. Within the initial burst, adsorbed or entrapped INN molecules are released into the media due to a high dissolution rate of the polymer surface coatings. Subsequent slower release was due to interaction of INN to hydrophilic chains of CS core, which was densified by ionic cross-linkers. According to Table S4 and Fig. 7a, there was no statistical difference between release rates of aPENs and bPENs at two pHs of media after $72 \mathrm{~h}$. Therefore, the introduction of these cross-linkers might cause $\mathrm{pH}$-independent drug release by preserving the stability of PENs through inter/intramolecular forces and thus decreasing total release.

To better understand the functions and behaviors of cross-linkers, drug release characteristics of single-layer and multilayer nanostructures were separately compared. As shown in Fig. 7b and Table S4, the lowest release rates were observed in aPENs and bPENs after the addition of DS. 
Although the strong repulsion between DS chains led to less compact structures, the particles could preserve the remaining drug mass inside and inhibit rapid outward diffusion. This phenomenon can be ascribed to $\mathrm{pKa}<2$ of DS, which behaves as a $\mathrm{pH}$ insensitive cross-linker, and the $\mathrm{pH}$ of media does not affect the number of charged ions much.

(a)

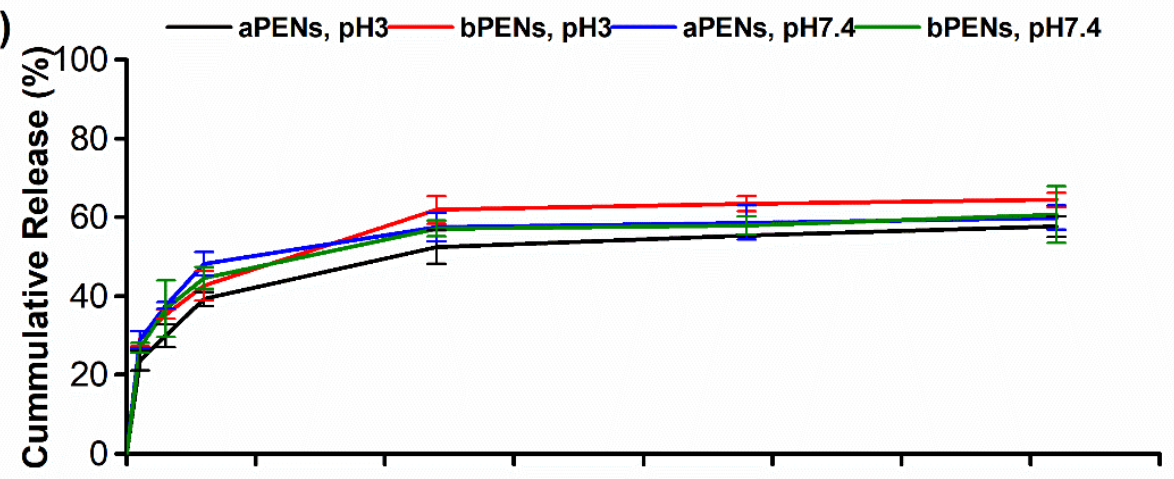

(b)

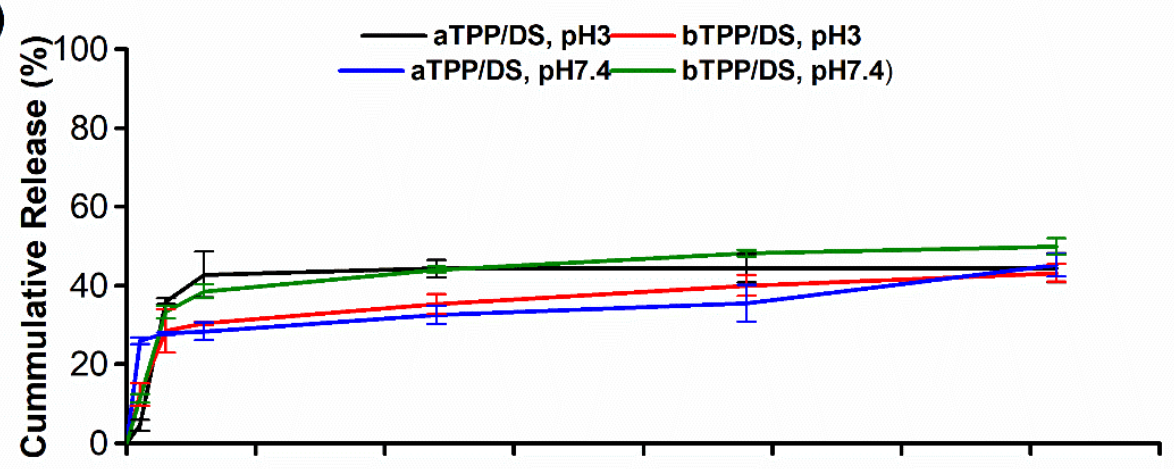

(c)

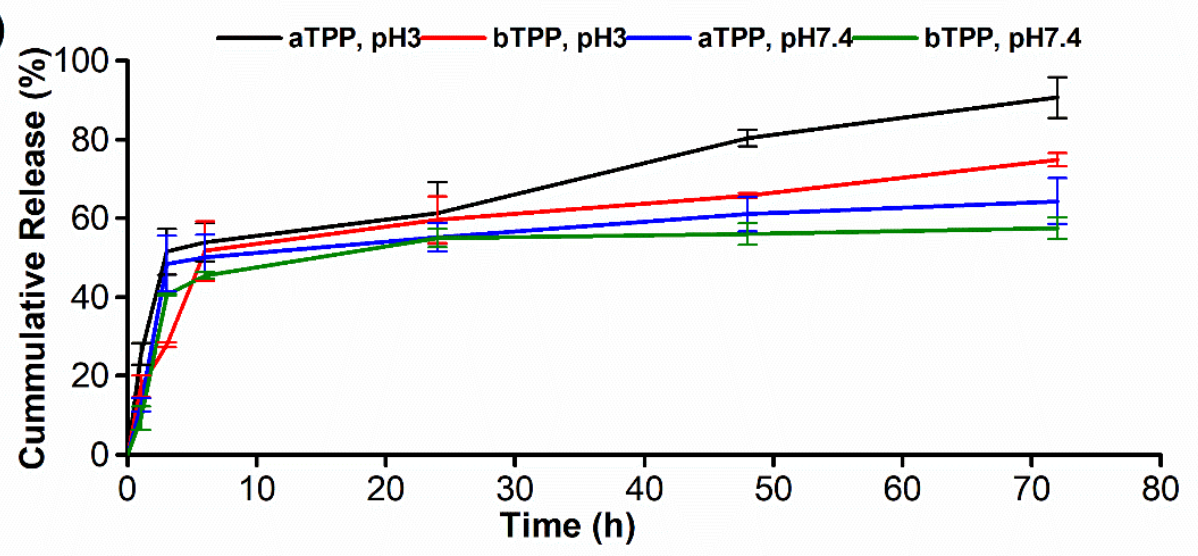


Fig. 7. In vitro release profiles of INN loaded PENs in three constructions at two pHs of release media (7.4 and 3); (a) PENs, (b) nanoparticles structured by two cross-linkers TPP and DS and (c) nanoparticles structured by TPP.

The other important cross-linker is TPP with pH-responsive behavior. As shown in Table S4 and Fig. 7c, single-layer particles showed higher release rates at $\mathrm{pH} 3,90.61 \pm 5.25 \%$ (aPENs) and $74.82 \pm 1.66 \%$ (bPENs), than pH 7.4, 64.32 $\pm 5.85 \%$ (aPENs) and $57.44 \pm 2.76 \%$ (bPENs). aPENs with single-layer structures showed significantly higher release rates than multilayer analogues at $\mathrm{pH} \mathrm{3,} \mathrm{and} \mathrm{the} \mathrm{presence} \mathrm{of} \mathrm{multiple} \mathrm{cross-linkers} \mathrm{led} \mathrm{to} \mathrm{significantly} \mathrm{decreased} \mathrm{release} \mathrm{rate}$ $(43.23 \pm 2.27 \%)$ at $\mathrm{pH}$ 7.4. It was expected that the extension of fully protonated CS chains with high amino-binding sites for TPP resulted in more compact structures and lowering release rate (Mattu, Li, \& Ciardelli, 2013), but low pH and insufficient TPP density affected nanoparticle dissociation, swelling and consequently increasing release rate. In bPENs, single-layer and multilayer nanostructures exhibited no significant different release rates except between TPP and TPP/DS at pH 3 (Table S4). It can be discussed by sufficient amino-binding sites of CS for TPP dissociated into $\mathrm{OH}^{-}, \mathrm{HP}_{3} \mathrm{O}_{10}{ }^{4-}$ and $\mathrm{P}_{3} \mathrm{O}_{10}{ }^{5-}$, which induces lower release rates.

The drug interaction to the particle is another factor which affects ionic interactions between the protonated amino groups of CS and negatively charged phosphoric groups. INN is neutrally charged due to pKa 4.75 in aPENs, and hydrogen bonding interactions probably mediate drug release. Nonetheless, the dominant interaction of INN in bPENs will be electrostatic interactions, which restrict INN diffusion and show a sustained release at different $\mathrm{pHs}$. Overall, these results suggest that the presence of multiple ionic cross-linkers had a significant influence on release profiles of the present structures, which are suitable in $\mathrm{pH}$-insensitive drug delivery systems.

\subsection{Kinetic study and release models of INN}

In order to gain insight into the INN release mechanism, different kinetic models were utilized to obtain information about the mass transport mechanisms in these nanosystems. The kinetics profiles of PENs were analyzed through zero-order, first-order, Higuchi, Hixson-Crowell, and Korsmeyer-Peppas models shown in Table 3. The best-fitting model was selected on the correlation coefficient value $\left(R^{2}\right)$ of the relationship between INN release and time. Considering the $\mathrm{R}^{2}$ values, the Korsmeyer-Peppas model was the best fit model for both PENs. In this model, the release mechanism of drug was governed by the value of release exponent, $\mathrm{n}$, in which $\mathrm{n} \leq 0.45$ 
corresponds to a Fickian diffusion mechanism, $0.45<\mathrm{n}<0.89$ to non-Fickian transport, $\mathrm{n}=0.89$ to Case II (relaxational) transport, and n > 0.89 to super case II transport (Dash, Murthy, Nath, \& Chowdhury, 2010). Herein, the value of $n \sim 0.2$ indicates that the INN transport mechanism is controlled by Fickian diffusion through non-swellable matrix diffusion kinetics (Barbosa, Costa Lima, \& Reis, 2019). Therefore, the release of INN from PENs is governed significantly by a diffusion process without the disintegration of the nanoparticles independent to $\mathrm{pH}$ of the crosslinkers and release media.

Table 3. INN kinetic models from aPENs and bPENs at two different pHs of media.

\begin{tabular}{|c|c|c|c|c|c|c|}
\hline & \multicolumn{6}{|c|}{ Correlation coefficient $\left(R^{2}\right)$} \\
\hline & $\begin{array}{l}\text { Zero-order } \\
(\mathrm{Qt}=\mathrm{Qo}+\mathrm{Kot})\end{array}$ & $\begin{array}{l}\text { First-order } \\
(\operatorname{lnQ} t=\operatorname{lnQ} 0+K 1 t)\end{array}$ & $\begin{array}{l}\text { Higuchi } \\
\left(\mathrm{Qt}=\mathrm{KHt}^{1 / 2}\right)\end{array}$ & $\begin{array}{l}\text { Hixson-Crowell's } \\
\sqrt[3]{W o}-\sqrt[3]{W t}=K s t\end{array}$ & $\begin{array}{l}\text { Korsmeyer- } \\
\text { Peppas's } \\
\left(\mathrm{Mt} / \mathrm{M}=\mathrm{Kt}^{\mathrm{n}}\right)\end{array}$ & $\mathrm{n}$ \\
\hline aPENs, pH3 & 0.757 & 0.686 & 0.896 & 0.710 & 0.969 & 0.214 \\
\hline bPENs, pH3 & 0.735 & 0.693 & 0.881 & 0.708 & 0.968 & 0.200 \\
\hline aPENs, pH7.4 & 0.640 & 0.579 & 0.801 & 0.600 & 0.921 & 0.169 \\
\hline bPENs, pH7.4 & 0.697 & 0.623 & 0.848 & 0.649 & 0.946 & 0.186 \\
\hline
\end{tabular}

\section{Conclusions}

Novel stable PENs were synthesized in the presence of multiple ionic cross-linkers, i.e., TPP and DS at pH 3 (aPENs) and 8 (bPENs). These spherical core/shell nanostructures were clearly visible in bPENs, but aPENs showed more compact structures with an indistinguishable shell. Evaluation of hydrodynamic size at the predetermined time intervals proved that the colloidal stability of bPENs was salt concentration-dependent; more specifically, higher salt concentration led to more stabilization. The release data also confirmed that both PENs demonstrated sustained release at different $\mathrm{pHs}$ of the release media; in fact, the release of INN from PENs is governed significantly by a diffusion process without disintegration of the nanoparticles in a $\mathrm{pH}$-independent manner. Overall, cross-linker $\mathrm{pH}$ was the main factor influencing particle stabilization, because bPENs demonstrated significantly higher stability than aPENs after 2 months' storage in PBS 10mM. 
These results lead us to offer a promising construct in this report for obtaining desirable stability of PENs and prolonged systemic circulation for a $\mathrm{pH}$-independent drug delivery system.

\section{Acknowledgments}

We gratefully acknowledge that this work was co-founded by the Ministry of Education, Youth, and Sports of the Czech Republic (Grant No. LO 1504). 


\section{References}

Ajun, W., Yan, S., Li, G., \& Huili, L. (2009). Preparation of aspirin and probucol in combination loaded chitosan nanoparticles and in vitro release study. Carbohydrate Polymers, 75(4), 566-574.

Bal, S. M., Slütter, B., van Riet, E., Kruithof, A. C., Ding, Z., Kersten, G. F., . . Bouwstra, J. A. (2010). Efficient induction of immune responses through intradermal vaccination with $\mathrm{N}$-trimethyl chitosan containing antigen formulations. Journal of Controlled Release, 142(3), 374-383.

Barbosa, A. I., Costa Lima, S. A., \& Reis, S. (2019). Application of pH-responsive fucoidan/chitosan nanoparticles to improve oral quercetin delivery. Molecules, 24(2), 346.

Can, H. K., Kavlak, S., ParviziKhosroshahi, S., \& Güner, A. (2018). Preparation, characterization and dynamical mechanical properties of dextran-coated iron oxide nanoparticles (DIONPs). Artificial cells, nanomedicine, and biotechnology, 46(2), 421-431.

Chavan, C., Bala, P., Pal, K., \& Kale, S. (2017). Cross-linked chitosan-dextran sulphate vehicle system for controlled release of ciprofloxaxin drug: An ophthalmic application. OpenNano, 2, 28-36.

Costalat, M., David, L., \& Delair, T. (2014). Reversible controlled assembly of chitosan and dextran sulfate: A new method for nanoparticle elaboration. Carbohydrate Polymers, 102, 717-726.

Dash, S., Murthy, P. N., Nath, L., \& Chowdhury, P. (2010). Kinetic modeling on drug release from controlled drug delivery systems. Acta Pol Pharm, 67(3), 217-223.

Diop, M., Auberval, N., Viciglio, A., Langlois, A., Bietiger, W., Mura, C., . . Zhao, M. (2015). Design, characterisation, and bioefficiency of insulin-chitosan nanoparticles after stabilisation by freezedrying or cross-linking. International journal of pharmaceutics, 491(1), 402-408.

Fan, W., Yan, W., Xu, Z., \& Ni, H. (2012). Formation mechanism of monodisperse, low molecular weight chitosan nanoparticles by ionic gelation technique. Colloids and Surfaces B: Biointerfaces, 90, 2127.

Gonçalves, E. M., \& da Piedade, M. E. M. (2012). Solubility of nicotinic acid in water, ethanol, acetone, diethyl ether, acetonitrile, and dimethyl sulfoxide. The Journal of Chemical Thermodynamics, 47, 362-371.

Hansson, A., Di Francesco, T., Falson, F., Rousselle, P., Jordan, O., \& Borchard, G. (2012). Preparation and evaluation of nanoparticles for directed tissue engineering. International journal of pharmaceutics, 439(1-2), 73-80.

Jonassen, H., Kjøniksen, A.-L., \& Hiorth, M. (2012). Stability of chitosan nanoparticles cross-linked with tripolyphosphate. Biomacromolecules, 13(11), 3747-3756.

Kishor, R., \& Ghoshal, A. K. (2016). Polyethylenimine Functionalized As-Synthesized KIT-6 Adsorbent for Highly CO2/N2 Selective Separation. Energy \& Fuels, 30(11), 9635-9644. 
Lin, Y.-H., Chung, C.-K., Chen, C.-T., Liang, H.-F., Chen, S.-C., \& Sung, H.-W. (2005). Preparation of nanoparticles composed of chitosan/poly- $\gamma$-glutamic acid and evaluation of their permeability through Caco-2 cells. Biomacromolecules, 6(2), 1104-1112.

Liu, L., Zheng, M., Librizzi, D., Renette, T., Merkel, O. M., \& Kissel, T. (2015). Efficient and Tumor Targeted siRNA Delivery by Polyethylenimine-graft-polycaprolactone-block-poly (ethylene glycol)-folate (PEI-PCL-PEG-Fol). Molecular pharmaceutics, 13(1), 134-143.

Mattu, C., Li, R., \& Ciardelli, G. (2013). Chitosan nanoparticles as therapeutic protein nanocarriers: The effect of ph on particle formation and encapsulation efficiency. Polymer composites, 34(9), 15381545.

Motiei, M., \& Kashanian, S. (2017). Novel amphiphilic chitosan nanocarriers for sustained oral delivery of hydrophobic drugs. European Journal of Pharmaceutical Sciences, 99, 285-291.

Motiei, M., Kashanian, S., Lucia, L. A., \& Khazaei, M. (2017). Intrinsic parameters for the synthesis and tuned properties of amphiphilic chitosan drug delivery nanocarriers. Journal of Controlled Release, $260,213-225$.

Motiei, M., Kashanian, S., \& Taherpour, A. (2017). Hydrophobic amino acids grafted onto chitosan: a novel amphiphilic chitosan nanocarrier for hydrophobic drugs. Drug development and industrial pharmacy, 43(1), 1-11.

Nikolić, G. S., Cakić, M. D., Glišić, S., Cvetković, D. J., Mitić, Ž. J., \& Marković, D. Z. (2017). Study of Green Nanoparticles and Biocomplexes Based on Exopolysaccharide by Modern Fourier Transform Spectroscopy. In Fourier Transforms-High-tech Application and Current Trends: InTech

Quiñones, J. P., Peniche, H., \& Peniche, C. (2018). Chitosan based self-assembled nanoparticles in drug delivery. Polymers, 10(3), 235.

Rampino, A., Borgogna, M., Blasi, P., Bellich, B., \& Cesàro, A. (2013). Chitosan nanoparticles: preparation, size evolution and stability. International journal of pharmaceutics, 455(1-2), 219228.

Umerska, A., Paluch, K. J., Inkielewicz-Stępniak, I., Santos-Martinez, M. J., Corrigan, O. I., Medina, C., \& Tajber, L. (2012). Exploring the assembly process and properties of novel crosslinker-free hyaluronate-based polyelectrolyte complex nanocarriers. International journal of pharmaceutics, 436(1-2), 75-87.

Verheul, R. J., Slütter, B., Bal, S. M., Bouwstra, J. A., Jiskoot, W., \& Hennink, W. E. (2011). Covalently stabilized trimethyl chitosan-hyaluronic acid nanoparticles for nasal and intradermal vaccination. Journal of Controlled Release, 156(1), 46-52. 
Wang, F., Yang, Y., Ju, X., Udenigwe, C. C., \& He, R. (2018). Polyelectrolyte complex nanoparticles from chitosan and acylated rapeseed cruciferin protein for curcumin delivery. Journal of agricultural and food chemistry, 66(11), 2685-2693.

Wang, H., Yang, B., \& Sun, H. (2017). Pectin-Chitosan Polyelectrolyte Complex Nanoparticles for Encapsulation and Controlled Release of Nisin. American Journal of Polymer Science and Technology, 3(5), 82.

Wang, W., Li, W., Wang, J., Hu, Q., Balk, M., Bieback, K., . . Lendlein, A. (2017). Folate receptor mediated genetic modification of human mesenchymal stem cells via folic acid-polyethyleniminegrafted poly (N-3-hydroxypropyl) aspartamide. Clinical hemorheology and microcirculation(Preprint), 1-17.

Wu, D., \& Delair, T. (2015). Stabilization of chitosan/hyaluronan colloidal polyelectrolyte complexes in physiological conditions. Carbohydrate Polymers, 119, 149-158.

Yudovin-Farber, I., Beyth, N., Weiss, E. I., \& Domb, A. J. (2010). Antibacterial effect of composite resins containing quaternary ammonium polyethyleneimine nanoparticles. Journal of Nanoparticle Research, 12(2), 591-603. 